\title{
The relationship between sensory processing sensitivity and psychological distress: A model of underpinning mechanisms and an analysis of therapeutic possibilities
}

psykologisk.no/sp/2017/11/e15

In the highly sensitive person, cognitive reactivity might be a driving mechanism in the relationship between sensory processing and psychological distress, write Hedda Bratholm Wyller and colleagues.

BY: Hedda Bratholm Wyller, Vegard Bruun Bratholm Wyller, Catherine Crane and Bergljot Gjelsvik

The concepts of sensory processing sensitivity (SPS) and the highly sensitive person (HSP) were introduced to the public and academic circles about two decades ago (Aron, 1996; Aron \& Aron, 1997).

SPS is described as a genetically-based personality trait involving hypervigilance to subtle changes in sensory signals and a tendency to be more easily overwhelmed when in a highly stimulating environment (Aron, Aron, \& Jagiellowicz, 2012). SPS is characterised by increased emotional reactivity, leading to attenuated cognitive functioning and problems in effectively selecting and deselecting stimuli. Initially, the trait was presented as dichotomous (either present or absent) and proposed to be present in about $15-20 \%$ of the population. This percentage - the HSPs - are said to be more easily affected than non-HSPs by a wide range of different sensory stimuli (e.g., loud noise, changes in temperature) and to suffer from impoverished top-down processing (Aron \& Aron, 1997).

The validity of the SPS construct has been questioned. While some claim that SPS captures individual differences not satisfactorily described by existing personality constructs (Aron \& Aron, 1997), others might argue that the conceptual framework draws on a comprehensive lineage of concepts depicting individual differences in sensitivity, such as introversion (Jung, 1923); brain stem arousal (Eysenck, 1967); low sensory threshold and social withdrawal (Chess \& Thomas, 1977); and introversion, neuroticism, and openness (Costa \& McCrae, 1992). Furthermore, questionnaire studies attempting to operationalise the SPS construct have yielded different dimensionalities. Nevertheless, studies suggest that SPS relates to aspects of biological functioning. There has been reported an association between SPS and genetic markers (the serotonin transporter gene 5-HTTLPR) (Licht, Mortensen, \& Knudsen, 2011), as well as between SPS and brain sensory processing assessed by imaging techniques (Gerstenberg, 2012; Jagiellowicz, Xu, Aron, et 
al., 2011). Also, several studies have indicated associations between SPS and psychological distress (Liss, Mailloux, \& Erchull, 2008; Hofmann \& Bitran, 2007).

One striking point in the SPS literature is that neither heterogeneity within the HSP category nor variation in individual sensitivity over time is considered. While the cut-off in the distribution (Aron \& Aron, 1997) gives empirical evidence for a pragmatic distinction between HSPs and non-HSPs, this observation should be distinguished from the assumption that SPS is fundamentally dichotomous. Rather, the trait might be more in line with continuum models in personality trait research (Costa \& McCrae, 1992). Furthermore, sensitivity might not be regarded as a static concept, but rather as a dynamic one which varies depending on a range of different factors within the individual (e.g., social context, sensory stimuli, circadian rhythms). Finally, for therapeutic interventions to have impact, parts of the SPS concept must be malleable. Developing a treatment for psychological problems associated with SPS would make little sense if the problems did not have plastic features.

Although several studies have confirmed an association between SPS and psychological distress, theoretical models attempting to explain this association are lacking. Such models are prerequisites, however, for developing methods to alleviate SPS-related psychological distress ( Clark, 2004). Identifying key mechanisms which drive problem maintenance and developing clinical interventions which are logically derived from such risk mechanisms has, therefore, clinical as well as theoretical interest.

The aim of this paper is twofold. Firstly, we present and discuss a model in which a cognitive science account of recurrent depression - the Differential Activation framework (Lau, Segal, \& Williams, 2004; Teasdale, 1988) - is applied on the relationship between SPS and psychological distress. Secondly, we discuss the clinical implications of this model. Drawing from advances in depression research (Lau et al., 2004; Teasdale, 1988), we start out by suggesting that it is the tendency to react to psychological problems, typically trying to change or get rid of them, which serves to maintain and exacerbate psychological distress in SPS. Thus, we argue that it is not SPS per se that should be regarded as psychologically inexpedient but rather the subsequent cognitive processes associated with the experiences conferred by the (assumed) trait. Irrespective of the extent to which SPS itself may be malleable, the hypothesised cognitive reactivity to sensory and emotional experience in those with SPS might represent a possible clinical target. A therapeutic intervention that specifically addresses cognitive reactivity is Mindfulness-Based Cognitive Therapy (MBCT; 
Segal, Williams, \& Teasdale, 2012). MBCT aims to alter negative thought processing through non-judgmental awareness and acceptance, thereby changing the relationship to the experience. Whilst MBCT was originally developed to target cognitive reactivity in depressed patients, we here discuss the possible therapeutic effects of it on SPS-related psychological distress.

\section{Sensory processing sensitivity and psychological} distress: A proposed model

\section{Associations between sensory processing sensitivity and psychological distress}

SPS has been found to correlate with a wide range of psychological problems, such as general anxiety/panic, tension and worry about everyday events, and social phobia (Neal, Edelmann, \& Glachan, 2002); harm avoidance and agoraphobic avoidance ( Bitran, 2007); and depression (Liss, Mailloux, \& Erchull, 2008). Corroborating these results, Benham (2006) found that SPS correlated with a wide range of physical health problems (Benham, 2006). This study is interesting as it suggests a possible link between SPS and conditions that are often understood as "functional" or "psychosomatic" in the medical literature. A possible explanation for the positive relationship between SPS and physical complaints is that HSPs are more sensitive to and aware of somatic sensations, paying attention to minor physiological sensations which others may not notice. This interpretation is in line with the hypervigilance model of pain perception (McDermid, Rollman, \& McCain, 1996) which suggests that chronic pain patients have a heightened sensitivity to pain (e.g., low threshold and tolerance). Such sensitivity, the model proposes, stems from increased attention to external stimulation and a preoccupation with pain sensations.

Evers and colleagues (2008) report that Ease of Excitation and Low Sensory Threshold (two sub-constructs of SPS) correlated negatively with sense of coherence, defined as a set of properties that are health promoting (e.g., the ability to understand, explain, and articulate the impact of stressors one is exposed to), and correlated positively with alienation and negative affectivity (Evers, Rasche, \& Schabracq, 2008). Accordingly, Liss and colleagues (2008) found that the same two factors correlated positively with poorer social skills, greater attention to details, and poorer communication (Liss et al., 2008), which might be mechanisms that increase the risk of psychological distress. Other studies confirm an association between SPS and different aspects of communication difficulties, such as the tendency to experience anxiety when communicating or thinking about communicating with others (Gearhart \& Bodie, 2012) and communication adaptability (Glonek. Nash, Shields, et al., 2007). Furthermore, SPS correlates with 
avoidance of social contact (Meyer, Ajchenbrenner, \& Bowles, 2005; Meyer \& Carver, 2000; Hofmann \& Bitran, 2007). Meyer and Carver (2000) found that SPS was associated with avoidant personality disorder, described as an intense desire for interpersonal affiliation coupled with persistent fear of social contact and pervasive social avoidance, isolation, and withdrawal (Meyer \& Carver, 2000).

In sum, SPS correlates with various mental disorders but also with negative psychological phenomena (such as emotional problems and low sense of coherence) and physical complaints. In this paper, we use the term psychological distress in an attempt to capture all these negative psychological conditions, not only those that meet diagnostic criteria. Furthermore, we argue that the evidence referred to above immediately suggest that a transdiagnostic perspective (i.e., an understanding of psychological conditions outside the conceptual structure of diagnosis) (Clark, 2009) should be incorporated in a model for the relationship between SPS and psychological distress.

\section{A model for psychological distress in HSPs}

The mechanisms underlying the association between SPS and psychological distress are poorly understood, and no comprehensive theoretical model has been presented so far. However, emotional regulation strategies (i.e., the ability to adjust the intensity and duration of one's own emotional reactions) have been found to mediate the relationship between SPS and depression, anxiety, and stress symptoms (Brindle, Moulding, Bakker et. al., 2015). Brindle and colleagues (2015) suggest that repeatedly experiencing aversive sensory states among HSPs impacts the general awareness and acceptance of internal states and the confidence that one can regulate them, in turn causing negative emotions. (Brindle et al., 2015). Thus, these researchers underline the possible importance of cognitive processes. Accordingly, we argue that a cognitive perspective might provide a clue for the mechanisms causing psychological distress in HSPs.

The concept of cognitive reactivity refers to both dysfunctional thought content and maladaptive thought processes (e.g., rumination, suppression, avoidance) (Teasdale, 1988). In psychological models of depression development, cognitive reactivity describes the activation of latent negative content and negative processing biases in response to transient low mood, which together serve to further escalate the negative affect (Teasdale, 1988). In addition to models of depression, cognitive reactivity has been suggested as an important transdiagnostic factor, especially for affective disorders (Drost, Van der Does, van Hemert, et al., 2014). We argue that cognitive reactivity is especially relevant 
in understanding the link between SPS and psychological distress because it emphasises that initial bottom-up reactivity to stimuli is bidirectionally linked to top-down processes. While the enhanced sensory-perceptual sensitivity to environmental stimuli might be hardwired and difficult to modify, cognitive reactivity, as a secondary reaction, is potentially malleable.

A

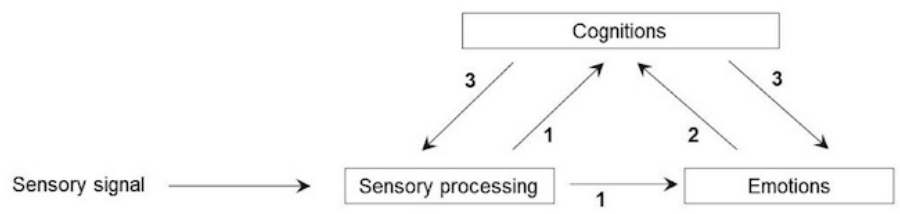

B



FIGURE 1: Simplified outline of the proposed model explaining the association between SPS and psychological distress and the possible beneficial effect of MBCT.

PANEL A: Normally, the processing of sensory signals gives rise to emotions and cognitions (1). Also, emotions in themselves impact on cognitive processes (2). Cognitions, in turn, feed back and modify both sensory processing and emotions (3).

PANEL B: According to the SPS concept, the HSP trait implies that a given sensory signal is amplified, in turn causing a general amplification of the signal transduction in the system. Thus, an aversive sensory signal (such as pain) will cause a stronger negative emotion in a HSP as compared to a non-HSP. Also, a sensory signal that is not aversive to a non-HSP might, because of its amplification, be aversive to an HSP (not shown in the figure). We suggest that the strong negative emotion and/or the strong sensory signal in itself might enact the phenomenon of cognitive reactivity, which in turn feeds back on the emotion as well as the sensory processing, setting up two vicious circles. Circle \#1 is responsible for emotional distress, such as depression and anxiety, whereas circle \#2 is responsible for distress related to somatic symptoms. These vicious circles, in turn, might have negative consequences for behaviour, personality, etc (not shown). MBCT might have transdiagnostic beneficial effects because this therapeutic approach addresses the negative processing of cognitive reactivity and thereby interferes with both vicious circles.

A simplified graphical depiction of the relationships among sensory processing, emotions, and cognitions is given in Figure 1 (Panel A). According to the SPS theory, the HSP trait implies that a given sensory signal is amplified, in turn causing a general amplification of the signal transduction in the system. Thus, an aversive sensory signal (such as pain) will cause a stronger negative emotion in an HSP as compared to a non-HSP (Panel B). The negative emotion enacts cognitive reactivity, which in turn feeds back on the emotion, setting up a vicious circle (Panel B, label 1). Alternatively, cognitive 
processes might be influenced directly from the amplified sensory signal, but the end result might be similar: increased negative emotionality as well as negative cognitive content and processes creating psychological distress. Thus, the concept of cognitive reactivity entails a shift in focus from external stressors towards internal, negative biases in processing (Lau et al., 2004) and might explain why psychological distress can be activated by subtle internal changes in HSPs, even when they are not exposed to environmental stimuli.

Taken together, the proposed model suggests that it is neither intense sensory information per se nor the related negative emotions which cause psychological distress to persist, so much as it is the subsequent, secondary cognitive reactions to them. These secondary reactions might be what distinguishes healthy and unhealthy HSPs. Moreover, they provide a unifying explanation for the associations between SPS and emotional distress such as depression and anxiety, somatic symptoms such as chronic pain and fatigue, and ensuing difficulties related to behaviour and personality.

Lisa (33 years old) often feels overwhelmed by noise and light and environments with high levels of sensory stimuli. Her emotions feel intense, and she finds herself preoccupied with her own sensory experiences and how she can manage them. She feels like she experiences situations at a "higher volume» than others. At times, her response is overwhelming, to the extent that she chooses to avoid situations which she finds difficult. However this avoidance has negative consequences and she ruminates about how sensory sensitivity affects her life. Sometimes the level of stimulation she feels means she becomes irritable and short-tempered and she regrets the negative impact that the sensitivity has on her social interactions. She feels as though other people don't understand properly how she experiences the world and that she can't control her own experience. As a consequence, she feels isolated and depressed. She wishes that she could find a way to deal better with her response. It is possible that learning mindfulness might have benefits for someone like Lisa in a number of areas. For example, mindfulness might enable Lisa to recognise early signs of becoming overwhelmed by her environment and to take action to reduce the level of stimulation. That way, rather than avoiding interactions altogether, she can better calibrate her functioning in the world. It might also help her develop metacognitive awareness to decipher when she is catastrophising about her sensitivity and thus potentially short-circuit the cognitive reactivity. Finally, the emphasis on acceptance and self-compassion might also help Lisa feel less isolated and less identified with the problems caused by her sensitivity. [This case is hypothetical and used for didactic 
purposes only.]

Although the proposed model is based on existing models of disease mechanisms in depression (Lau et al., 2004; Teasdale, 1988), it has important novel aspects. Originally, cognitive reactivity describes a process activated in the context of low mood, but we argue that in the case of HSPs, amplified sensory processing is enough to activate cognitive reactivity, independent of mood. Also, we emphasise that for HSPs, the model is transdiagnostic (i.e., it applies to SPS-related psychological distress regardless of diagnostic categorization). In addition, our model entails an original perspective on the associations between SPS and physical complaints that are often labelled "psychosomatic." For instance, distress such as chronic pain and chronic fatigue might be explained as an amplification of sensory signals due to cognitive reactivity (Panel B, label 2).

Our model also bears resemblance with the metacognitive model of emotional disorder (Wells \& Matthews, 1994) in which activation of a particular style of thinking, called the Cognitive-Attentional Syndrome (CAS), is seen as important in the maintenance and recurrence of depression. CAS shares important similarities with cognitive reactivity, and both concepts emphasise the importance of metacognition (i.e., cognition about cognition) in maintaining disease mechanisms. However, our model differs from metacognitive models of emotional disorders in that it relates to sensory processing. As a result, it links cognitive processes to embodied experiences which is a core element of the SPS trait. Thus, we argue that our model has advantages compared to existing models when explaining SPSassociated psychological distress.

The proposed model is supported in part by research into conditions characterised by strong physical sensations, such as chronic fatigue syndrome (CFS) and anxiety. In CFS, evidence suggests that greater acceptance of fatigue symptoms is associated with lower levels of fatigue (Van Damme, Crombez, Van Houdenhove, et al., 2006). In anxiety, people who meet their unpleasant emotions with acceptance instead of trying to get rid of them experience a decreased heart rate compared to people who suppress their emotions (Campbell-Sills, Barlow, Brown, et al., 2006).

If cognitive reactivity is a main determinant of psychological distress in HSPs, then a clinical intervention which targets this process may be efficacious. Based on the proposed model, we argue that what HSPs need is to increase their capacity to short-circuit their selfperpetuating vicious cycles. For this purpose, Mindfulness-Based Cognitive Therapy (MBCT; Segal et al., 2012) emerges as a therapeutic possibility. 


\section{Mindfulness-Based Cognitive Therapy: A potential intervention for psychological distress in highly sensitive persons?}

Mindfulness has been described as awareness that arises through paying attention, on purpose, in the present moment, nonjudgementally (Kabat-Zinn, 1990). Mindfulness meditation aims to train the capacity to observe thoughts, feelings and physiological sensations, irrespective of content, without having to avoid them or automatically let them determine one's actions.

\section{Mindfulness-Based Cognitive Therapy (MBCT)}

MBCT (Segal et al., 2012) combines meditation practices with psycho-educative elements from CBT. The 8-week programme forms part of a wider group of so-called 'third-wave' therapies such as Mindfulness-Based Stress Reduction (MBSR), Dialectical Behaviour Therapy (DBT) and Acceptance and Commitment Therapy (ACT). Unlike traditional Cognitive-Behavioural Therapy (CBT), there is no emphasis in MBCT and in other third-wave therapies on changing the content of cognitions. Rather, the emphasis is on changing one's relationship to experience.

MBCT is a cognitive science modification of MBSR, which means that it is based on a cognitive science account of how depression recurs and that it was specifically developed to prevent depressive relapses. Building on the Differential Activation Hypothesis (Lau et al., 2004), the theoretical premise in MBCT is that vulnerability to depressive relapse is partly due to individual differences in how easily low mood reactivates a pattern of negative cognitive content and maladaptive processing (i.e., cognitive reactivity). MBCT aims to short-circuit these relapse processes through non-judgmental awareness and acceptance of the negative thoughts that occur during transient states of low mood. This mindful state is hypothesised to enable individuals to respond to situations more reflectively rather than being caught up in maladaptive automatic patterns of thinking or behaviour.

There is robust evidence that MBCT prevents relapses in recurrent depression, and a recent meta-analysis showed that MBCT was particularly efficacious for those with more pronounced residual depression symptoms (Kuyken, Warren, Taylor, et al., 2016).

The potential merit of Mindfulness-Based Cognitive Therapy for

\section{psychological distress in highly sensitive persons}

We suggest that applying MBCT to SPS-related psychological distress might have merit. That is because the therapeutic approach addresses cognitive reactivity which we propose is also a pivotal mechanism in SPS-related psychological distress. Furthermore, MBCT might have transdiagnostic beneficial effects in SPS since our 
model indicates that cognitive reactivity is the driving force for both emotional distress and distress related to somatic symptoms (Figure 1, Panel B). Of note, the purpose of MBCT would not be to alter the SPS-trait per se but rather to ameliorate the psychological distress that occurs more frequently in HSPs.

Interestingly, several studies have found that mindfulness training increases perceptual sensitivity (e.g., MacLean, Ferrer, Aichele, et al., 2010). As such, one might question whether further increases in sensitivity would benefit HSPs. That is because being overly aware of subtle sensory changes might compound hypervigilance and rumination (Cioffi, 1991), problems that are assumed to be central in SPS. However, mindful processing is hypothesised to increase not just attention to sensory signals but also awareness of one's interpretation of such signals and one's response to them (Farb, Daubenmier, Price, et al., 2015). For instance, increased awareness of the bodily expression of an emotion might lead to greater awareness of one's own emotional life (Mehling, Gopisetty, Daubenmier, et al., 2009). A more spacious context for emotional experience is thereby created as opposed to taking emotions and their bodily expressions at face value.

Whilst there is a sound theoretical basis for a possible positive effect of mindfulness training on SPS-related psychological distress, empirical evidence is scarce. So far, only one study has investigated the effect of mindfulness training on SPS (Soons, Brouwers \& Tomic, 2010). Results indicated that Mindfulness-Based Stress Reduction (an intervention focusing on stress reduction and physical health conditions) had a large effect on stress, social anxiety, selfacceptance and personal growth, while the effect on emotional empathy and self-transcendence was more moderate (Soons et al., 2010). Further studies in this field are required, addressing HSPs' hypothesised problems of cognitive reactivity as well as the clinical effect of mindfulness-based interventions. One prediction from our model would be that experimentally-induced aversive sensations would increase cognitive reactivity more in HSPs as compared to non-HSPs controls. Cognitive reactivity could be measured by the Perseverative Thinking Questionnaire (PTQ; Ehring, Zetsche, Weidacker, et al., 2011), which is based on a transdiagnostic framework. Furthermore, the extent to which certain background factors (e.g., gender, childhood adversities, cultural background, genetic traits) influence cognitive reactivity in the HSP group is also a topic for further exploration. As for clinical research, the possible beneficial effect of MBCT on SPS-related psychological distress should be investigated in feasibility studies and, if promising, in subsequent randomised controlled trials.

Conclusion 
In this paper, we have argued that the association between SPS and psychological distress is not caused by SPS per se. Instead, by applying a model that was originally developed for recurrent depression, we suggest that a secondary phenomenon of cognitive reactivity (i.e., maladaptive cognitive content and processes) might be a driving mechanism in the relationship between SPS and psychological distress.

Based on this premise, a therapeutic intervention which targets cognitive reactivity might have merit for SPS-related psychological distress. MBCT was specifically developed to target cognitive reactivity and has documented effects on depression. Our proposed model of underlying mechanisms, as well as the possible beneficial effect of MBCT on SPS-related psychological distress, should be addressed in future research.

\section{References}

Aron, E. (1996). The highly sensitive person: How to thrive when the world overwhelms you. New York: Broadway Books.

Aron, E. N., \& Aron, A. (1997). Sensory-processing sensitivity and its relation to introversion and emotionality. Journal of Personality and Social Psychology, 73(2), 345-368. doi:10.1037/0022-3514.73.2.345

Aron, E. N., Aron, A., \& Jagiellowicz, J. (2012). Sensory processing sensitivity: a review in the light of the evolution of biological responsivity. Personality and Social Psychology Review, 16(3), 262282. doi:10.1177/1088868311434213

Benham, G. (2006). The highly sensitive person: Stress and physical symptom reports. Personality and Individual Differences, 40(7), 1433-1440. doi:10.1016/j.paid.2005.11.021

Brindle, K., Moulding, R., Bakker, K., \& Nedeljkovic, M. (2015). Is the relationship between sensory-processing sensitivity and negative affect mediated by emotional regulation? Australian Journal of Psychology, 67(4), 214-221. doi:10.1111/ajpy.12084

Campbell-Sills, L., Barlow, D. H., Brown, T. A., \& Hofmann, S. G. (2006). Effects of suppression and acceptance on emotional responses of individuals with anxiety and mood disorders. Behaviour Research and Therapy, 44(9), 1251-1263.

doi:10.1016/j.brat.2005.10.001

Chess, S., \& Thomas, A. (1977). Temperamental individuality from childhood to adolescence. Journal of the American Academy of Child Psychiatry, 16(2), 218-226. doi:10.1016/s00027138(09)60038-8 
Cioffi, D. (1991). Beyond attentional strategies: Cognitive-perceptual model of somatic interpretation. Psychological Bulletin, 109, 25-41. doi: $\underline{10.1037 / 0033-2909.109 .1 .25}$

Clark, D. M. (2004). Developing new treatments: On the interplay between theories, experimental science and clinical innovation. Behaviour Research and Therapy, 42(9), 1089-1104.

Clark, D. A. (2009). Cognitive behavioral therapy for anxiety and depression: Possibilities and limitations of a transdiagnostic perspective. Cognitive Behaviour Therapy, 38(S1), 29-34. doi: $\underline{10.1080 / 16506070902980745}$

Costa, P. T., \& McCrae, R. R. (1992). Revised NEO Personality Inventory (NEO PI-R) and NEO Five-Factor Inventory (NEO FFI): Professional manual: Psychological Assessment Resources.

Drost, J., Van der Does, W., van Hemert, A. M., Penninx, B. W., \& Spinhoven, P. (2014). Repetitive negative thinking as a transdiagnostic factor in depression and anxiety: A conceptual replication. Behaviour Research and Therapy, 63, 177-183. doi:10.1016/j.brat.2014.06.004

Ehring, T., Zetsche, U., Weidacker K., Wahl, K., Schönfeld, S., Ehlers, A. (2011). The Perseverative Thinking Questionnaire (PTQ): Validation of a content-independent measure of repetitive negative thinking. Journal of Behavior Therapy and Experimental Psychiatry, 42, 225-232. doi:10.1016/j.jbtep.2010.12.003

Evers, A., Rasche, J., \& Schabracq, M. J. (2008). High sensoryprocessing sensitivity at work. International Journal of Stress Management, 15(2), 189-198. doi:10.1037/1072-5245.15.2.189

Eysenck, H. J. (1967). The Biological Basis of Personality (Vol. 689). Piscataway, NJ: Transaction publishers.

Farb, N., Daubenmier, J., Price, C. J., Gard, T., Kerr, C., Dunn, B. D., ... \& Mehling, W. E. (2015). Interoception, contemplative practice, and health. Frontiers in Psychology, 6. doi:10.3389/fpsyg.2015.00763

Gearhart, C. C., \& Bodie, G. D. (2012). Sensory-processing sensitivity and communication apprehension: Dual influences on self-reported stress in a college student sample. Communication Reports, 25(1), 27-39. doi:10.1080/08934215.2012.672216

Gerstenberg, F. X. R. (2012). Sensory-processing sensitivity predicts performance on a visual search task followed by an increase in perceived stress. Personality and Individual Differences, 53(4), 496-500. doi:10.1016/j.paid.2012.04.019 
Glonek, K., Nash, E., Shields, V., Sawyer, C. R., \& Behnke, R. R. (2007, November). Communication adaptability as a function of nervous system mobility and sensory processing sensitivity. Paper presented at the meeting of the National Communication Association, Chicago, IL.

Hofmann, S. G., \& Bitran, S. (2007). Sensory-processing sensitivity in social anxiety disorder: Relationship to harm avoidance and diagnostic subtypes. Journal of Anxiety Disorders, 21(7), 944-954. doi:10.1016/j.janxdis.2006.12.003

Jagiellowicz, J., Xu, X., Aron, A., Aron, E., Cao, G., Feng, T., \& Weng, $X$. (2011). The trait of sensory processing sensitivity and neural responses to changes in visual scenes. Social Cognitive and Affective Neuroscience, 6(1), 38-47. doi:10.1093/scan/nsq001

Jung, C. G. (1923). Psychological Types or the Psychology of Individuation. New York: Harcourt, Brace.

Kabat-Zinn, J. (1990). Full catastrophe living: The program of the stress reduction clinic at the University of Massachusetts Medical Center. New York: Delta.

Kuyken, W., Warren, F. C., Taylor, R. S., Whalley, B., Crane, C., Bondolfi, G., ... \& Segal, Z. (2016). Efficacy of mindfulness-based cognitive therapy in prevention of depressive relapse: An individual patient data meta-analysis from randomized trials, JAMA Psychiatry, 73(6), 565-574. doi:10.1001/jamapsychiatry.2016.0076

Lau, M. A., Segal, Z. V., \& Williams, J. M. G. (2004). Teasdale's differential activation hypothesis: Implications for mechanisms of depressive relapse and suicidal behaviour. Behaviour Research and Therapy, 42(9), 1001-1017. doi:10.1016/j.brat.2004.03.003

Licht, C. L., Mortensen, E. L., \& Knudsen, G. M. (2011). Association between sensory processing sensitivity and the serotonin transporter polymorphism 5-HTTLPR short/short genotype. Biological Psychiatry, 69(9), 152s-153s.

Liss, M., Mailloux, J., \& Erchull, M. J. (2008). The relationships between sensory processing sensitivity, alexithymia, autism, depression, and anxiety. Personality and Individual Differences, 45(3), 255-259. doi:10.1016/j.paid.2008.04.009

MacLean, K. A., Ferrer, E., Aichele, S. R., Bridwell, D. A., Zanesco, A. P., Jacobs, T. L., ... \& Wallace, B. A. (2010). Intensive meditation training improves perceptual discrimination and sustained attention. Psychological Science, 21(6), 829-839.

doi: $\underline{10.1177 / 0956797610371339}$ 
McDermid, A. J., Rollman, G. B., \& McCain, G. A. (1996).

Generalized hypervigilance in fibromyalgia: Evidence of perceptual amplification. Pain, 66(2-3), 133-144. doi:10.1016/0304-

3959(96)03059-x

Mehling, W. E., Gopisetty, V., Daubenmier, J., Price, C. J., Hecht, F. M., \& Stewart, A. (2009). Body awareness: Construct and self-report measures. PLOS ONE, 4(5), e5614.

doi:10.1371/journal.pone.0005614

Meyer, B., Ajchenbrenner, M., \& Bowles, D. P. (2005). Sensory sensitivity, attachment experiences, and rejection responses among adults with borderline and avoidant features. Journal of Personality Disorders, 19(6), 641-658. doi:10.1521/pedi.2005.19.6.641

Meyer, B., \& Carver, C. S. (2000). Negative childhood accounts, sensitivity, and pessimism: A study of avoidant personality disorder features in college students. Journal of Personality Disorders, 14(3), 233-248. doi:10.1521/pedi.2000.14.3.233

Neal, J. A., Edelmann, R. J., \& Glachan, M. (2002). Behavioural inhibition and symptoms of anxiety and depression: Is there a specific relationship with social phobia? British Journal of Clinical Psychology, 41(Pt. 4), 361-374. doi:10.1348/014466502760387489

Segal, Z. V., Williams, J. M. G., \& Teasdale, J. D. (2012).

Mindfulness-Based Cognitive Therapy for depression. Guilford Press.

Soons, I., Brouwers, A., \& Tomic, W. (2010). An experimental study of the psychological impact of a Mindfulness-Based Stress Reduction Program on highly sensitive persons. Europe's Journal of Psychology, 6(4), 148-169. doi:10.5964/ejop.v6i4.228

Teasdale, J. D. (1988). Cognitive vulnerability to persistent depression. Cognition \& Emotion, 2(3), 247-274.

doi: $10.1080 / 02699938808410927$

Van Damme, S., Crombez, G., Van Houdenhove, B., Mariman, A., \& Michielsen, W. (2006). Well-being in patients with chronic fatigue syndrome: The role of acceptance. Journal of Psychosomatic Research, 61(5), 595-599. doi:10.1016/j.jpsychores.2006.04.015 Wells, A. \& Matthews, G. (1994). Attention and emotion: A clinical perspective. Hove: Erlbaum.

Citation

Wyller, H. B., Wyller, V. B. B., Crane, C., \& Gjelsvik, B. (2017). The relationship between sensory processing sensitivity and 
psychological distress: A model of underpinning mechanisms and an analysis of therapeutic possibilities. Scandinavian Psychologist, 4, e15. https://doi.org/10.15714/scandpsychol.4.e15

Abstract

The relationship between sensory processing sensitivity and psychological distress: A model of underpinning mechanisms and an analysis of therapeutic possibilities

Sensory processing sensitivity (SPS) is a recently coined concept describing a predisposition toward extensive processing of sensory stimuli. It has been conceptualised as an innate, dichotomous personality trait. SPS is associated with elevated psychological distress (mental disorders as well as emotional and somatic symptoms), but the underlying mechanisms are unknown and no specific treatment approaches exist. The aim of this paper is twofold. Firstly, building on contemporary theories of depression aetiology, we propose and discuss a theoretical model for the relationship between SPS and psychological distress. This model suggests that it is dysfunctional cognitive responses to aversive sensory and emotional stimuli (labelled cognitive reactivity) - not the stimuli in and of themselves - which serve to maintain and exacerbate psychological distress in SPS. Secondly, we discuss the clinical implications of the proposed model. Because cognitive reactivity is addressed in Mindfulness-Based Cognitive Therapy (MBCT), which has proven efficient at preventing depressive relapse, we suggest that MBCT might have clinical merit on SPS-related psychological distress as well. In conclusion, we suggest that future research should address the proposed model as well as the possible beneficial effect of MBCT for SPS-related psychological distress.

Keywords: highly sensitive person, mindfulness, mindfulness-based cognitive therapy, sensory processing sensitivity.

Author affiliations: Hedda Bratholm Wyller - Department of Psychology, University of Oslo, Oslo, Norway; Vegard Bruun Bratholm Wyller - Department of Pediatrics, Akershus University Hospital, Lørenskog, Norway; Catherine Crane - Department of Psychiatry, University of Oxford, Warneford Hospital, Oxford, UK; Bergljot Gjelsvik - Department of Psychology, University of Oslo, Oslo, Norway, \& Department of Psychiatry, University of Oxford, Warneford Hospital, Oxford, UK.

Contact information: Hedda Bratholm Wyller, Nordstrandv. 9, N-1170 Oslo. Email: heddabb@student.sv.uio.no.

Received: August 21, 2017. Accepted: October 25, 2017.

Published: November 23, 2017.

Language: English. 
Competing interests: The authors alone are responsible for the contents and writing of this paper. Dr. Catherine Crane is affiliated with the Oxford Mindfulness Centre; however, she does not receive any additional remuneration for any training workshops, books or presentations relating to mindfulness. Dr. Bergljot Gjelsvik is a trained teacher in MBCT and has taught MBCT in the last years as part of her programme of research; however, she does not receive any additional remuneration for any training workshops or presentations relating to mindfulness.

This is a peer-reviewed paper. 